Research Article

\title{
Hydromagnetic Flow of Prandtl Nanofluid Past Cylindrical Surface with Chemical Reaction and Convective Heat Transfer Aspects
}

\author{
Kottakkaran Sooppy Nisar, ${ }^{1}$ S. Bilal $\left(\mathbb{D},{ }^{2}\right.$ Imtiaz Ali Shah, ${ }^{2}$ M. Awais, ${ }^{3}$ Khalil-Ur-Rehman, ${ }^{2}$ \\ Ilyas Khan, ${ }^{4}$ and Phatiphat Thonthong ${ }^{5}$ \\ ${ }^{1}$ Department of Mathematics, College of Arts and Sciences, Prince Sattam bin Abdulaziz University, \\ Wadi Aldawaser 11991, Saudi Arabia \\ ${ }^{2}$ Department of Mathematics, Air University, PAF Complex E-9, Islamabad 44000, Pakistan \\ ${ }^{3}$ Department of Mathematics, Quaid-i-Azam University, Islamabad 44000, Pakistan \\ ${ }^{4}$ Department of Mathematics, College of Science Al-Zulfi, Majmaah University, Al-Majmaah 11952, Saudi Arabia \\ ${ }^{5}$ King Mongkut's University of Technology North Bangkok Renewable Energy Research Centre (RERC) Wongsawang, Bangsue, \\ Bangkok 10800, Thailand
}

Correspondence should be addressed to S. Bilal; smbilal@math.qau.edu.pk

Received 22 August 2020; Revised 9 October 2020; Accepted 16 November 2020; Published 19 January 2021

Academic Editor: Muhammad mubashir bhatti

Copyright (c) 2021 Kottakkaran Sooppy Nisar et al. This is an open access article distributed under the Creative Commons Attribution License, which permits unrestricted use, distribution, and reproduction in any medium, provided the original work is properly cited.

\begin{abstract}
Thermodynamical attributes of non-Newtonian fluids over stretched surfaces have gained pervasive essence due to extensive utilization in extruding plastic sheet procedures, liquid film condensation, glass blowing, paper production, biopolymer cylinder coatings, and so forth. So, currently communication is aimed to candidly explicate flow characteristic of Prandtl fluid generated by axial stretching of cylindrical surface. Mathematical modelling by using conservation laws of momentum, energy and concentration fields containing the aspects of magnetic field, convective heating, and chemical reaction are presented initially in the form of partial differential expressions. Later on, these attained PDEs are transmuted into nonlinear ordinary differential equations with implementation of similarity variables. Numerical approach renowned as shooting technique with improved coefficient of the Runge-Kutta (R-K) method by Cash and Karp is used to access accurate solution. Linear curved fitting analysis is also performed to analyze results. Influence of flow-controlling parameters on associated profiles is revealed through graphical visualization. Stream line plots representing flow behavior of Prandtl fluid versus different magnitudes of the curvature parameter are adorned. Variation in friction drag force at wall, heat flux, and concentration gradient are evaluated through numerical data and with interpolation of linear curved fittings. It is deduced from results that increasing curvature parameter momentum and temperature distributions enriches whereas skin-friction coefficient depicts reverse pattern. It is also inferred that temperature shows incrementing deviation in the absence of chemical reaction whereas concentration profiles exhibit reduction with consideration of influence of chemical reaction parameter. Magnetic field tends to reduce the velocity and create thinness of boundary layer thickness.
\end{abstract}

\section{Introduction}

Analysis of non-Newtonian fluids has attained superb attention of researchers due to numerous scientific implementations in multiple fields such as food mixing, multigrade oils, composition of materials, wire drawing, hot rolling, petroleum productions, metallurgical procedures, manufacturing of materials, preventive coating, lubricating products, polymerization processes, ink-jet printers, geophysical flows, liquid crystallizations, and several others. Taking into consideration the aforementioned extensive applicability in different technological and industrial 
frameworks, a lot of analysis has been conducted in this direction in recent years. However, the complexity about the non-Newtonian model is its mathematical modelling on behalf of different rheological features. Hence, there is no single constitutive mathematical model which is present, which expresses the fluid characteristics of all non-Newtonian fluids single handedly. Consequently, non-Newtonian fluid models are categorized into time-dependent, time-independent, viscoelastic, and viscoinelastic fluids. Among these subclasses, the most practicing fluids are viscoinelastic fluids. Dunn [1] presented pioneering investigation on the properties of viscoinelastic fluids and depicted behavior of strain of such fluids against application of stress. In the literature, a lot of fluid models are presented to elaborate characteristics of viscoinelastic fluids, but it is found that Prandtl fluid is the most fittest to explicate features of viscoinelastic fluids. Peristaltic movement of Prandtl fluid in endoscope was investigated by Nadeem et al. [2] by calculating perturbation solution of modelled problem with implementation of analytical approach. Akbar [3] considered blood as viscoinelastic fluid and delineated flow features in stenosed tapered arteries by computing perturbation solution. Jothi et al. [4] discussed the physical aspects of magnetically effected Prandtl fluid flow in a symmetric channel. Nazari et al. [5] analyzed convective heat transfer of Prandtl nanofluid flow saturated in lid-driven enclosure. Hossainy et al. [6] discussed the influence of yielding stress on magneto hydrodynamic boundary layer fluid flow of $3 \mathrm{D}$ Casson nanofluid over stretched surface entrenched in a porous medium. Bilal et al. [7] adumbrated flow features of the Prandtl fluid model over stretching sheet along with effectiveness of double-diffusive heating.

The mass transport phenomenon in many scientific disciplines involves molecular and convective transport of atoms and molecules. The driving force for producing such type of molecular diffusion is the concentration differences which can generate to application of chemical reaction within flow domain. The process of chemical reaction arises with addition of multiple dissimilar natured particles, and an outcome interaction of particles is produced. Mass transport procedures with effectiveness of chemical reactions have many practical applications, e.g., combustion system, metallurgy, distribution of fertilizers in agriculture field, nuclear reactor safety, and many more. In view of such extensive applications several interesting studies are conducted. For the sake of brevity, some of them are mentioned. Effect of chemical reactions on viscous fluid flow over horizontal plate was discussed by Anjalidevi and Kandasamy [8]. Zhang and Zheng [9] reported influence of chemical reactive species in thermosolutal Marangoni flow of electrified viscous fluid and predicted reduction in nanoparticle concentration with respect to uplift in chemical reaction magnitude. Chaudhary and Merkin [10] investigated thermophysical attributes of viscous fluid influenced by chemical reaction effects. Nandkeolyar et al. [11] described stagnant dissipative nanofluid in the attendance of chemically reaction and magnetic field. Hossainy and Eid [12] analyzed hydrothermal efficiency of non-Newtonian hybrid nanofluid in a heat-exchange channel by generation of chemical reactions in domain. Eid and Makinde [13] studied collaborative aspects of radiative heat energy and chemical reaction on electrically conducting nanofluid flow over a stretching sheet immersed in a permeable medium. The effects of slip velocity and chemical reaction generated in stagnant flow of nanofluid over a stretching sheet embedded in a porous medium were studied by Eid [14]. In precise, investigators are still working to explore hidden features of chemical reaction on Newtonian and non-Newtonian flows $[7,15,16]$.

In the today technological world, nanotechnology is considered one of momentous inventions which have brought advancement in industrial rebellion. Nanotechnology based on nanofluids is engineered colloidal suspension of nanometer-sized particles in host fluids. Before the discovery of nanoparticles, liquids with low thermophysical characteristics are capitalized in multiple procedures which produce deficiencies in output. So, Choi [17] proposed the idea of nanofluids by experimentally measuring their intrinsic features possessed by nanoparticles. Buongiorno [18] evaluated enhancement in thermal conductance of ordinary fluids with the induction of nanoparticles. Flow analysis of viscous fluid generated by an exponential stretching of surface by adding nanoparticles was probed by Nadeem et al. [19]. Reza et al. [20] interrogated influence of carbon nanotubes in peristaltic movement of water in a permeable channel under the impact of magnetic field. Peyghambarzadeh et al. [21] disclosed application of nanofluids in increasing automotive cooling. In recent years, extensive work on nanofluid flow analysis in numerical computational domains along with applications was manifest. So, for interest of readers and getting their motivation in this work few references are mentioned. Alaidrous and Eid [22] adumbrated nanofluid flow of nonNewtonian liquid under impedance of electromagnetic radiations and highly rated reactions. Eid and Mabood [23] irreversibility entropy generated aspects of carbon nanotube (CNT) placement in micropolar dusty fluid mixed with kerosene oil. The uplift in thermal characteristics of blood flow with induction of gold plated hybridized nanoparticles past a stretching sheet was analyzed by Eid [24]. Also, [25-30] presented the literature discusses by various researchers on nanoliquids.

Analysis of fluid flows under impression of magnetic field has been demanding research area among researchers due to overwhelming implications in industry, engineering, medical technology, and so many. In recent years, magneto hydrodynamics of electrically conducting flows has been investigated extensively in multiple physical configurations. Likewise, Lahmar et al. [31] examined transportation of an unsteady ferro magnetic nanofluid between two parallel plates by considering thermal heat conduction as a function of temperature in the presence of magnetic source. Some recent investigations in this direction are enclosed in $[32,33]$.

The abovementioned literature survey reflects prominence about thermophysical features of non-Newtonian fluid flow. However, it is seen that analysis of the Prandtl fluid model in the presence of chemical reaction and 
magnetic field over melted heating cylinder has not been scrutinized yet. So, current effort is made to fill this gap. In this regards, mathematical modelling of the non-Newtonian fluid rheological model with heat transfer aspects is attained in the form of partial differential equations by applying boundary layer approach. Afterwards, suitable scaling transformations are used for conversion into ordinary differential expressions. Improved numerical simulations by means of the Runge-Kutta method with improved coefficients presented by Cash and Karp are used. Computed results are presented through graphical and tabular manners.

\section{Mathematical Formulation}

Assume 2-D, in-compressed, time-independent, and electrified Prandtl nanoliquid persuaded by an axially stretchable cylinder. Let $v$ and $u$ be the velocity constituents along $r$ and $x$ directions, respectively, as shown in Figure 1 . The cylinder is stretched axially with linear velocity. The magnetic field of strength $B^{\circ}$ is applied perpendicular to cylinder axis. Furthermore, the generated magnetic field is very small in comparison with applied one due to low magnetic Reynolds number assumption.

Prandtl proposed the following rheological model [16]:

$$
\mathbf{S}=\left(\frac{A}{\dot{\gamma}} \sin ^{-1}\left(\frac{1}{c} \dot{\gamma}\right)\right) \mathbf{A}_{1},
$$

where fluid parameters are denoted with $A$ and $c, \mathbf{A}_{1}$ is first Rivlin-Ericksen tensor, and $\dot{\gamma}$ is defined as

$$
\dot{\gamma}=\sqrt{\frac{1}{2} \operatorname{tr} \mathbf{A}_{1}^{2}}
$$

By expanding $\sin ^{-1}(\cdot)$ function up to second-order approximation using Taylor's series,

$$
\sin ^{-1}\left(\frac{\dot{\gamma}}{c}\right)=\frac{\dot{\gamma}}{c}+\frac{\dot{\gamma}^{3}}{6 c^{3}}, \quad \text { where }\left|\frac{1}{c} \dot{\gamma}\right|<1 .
$$

By means of usual boundary layered approximations, the constitutive expressions become

$$
\begin{aligned}
\frac{\partial(r u)}{\partial x}+\frac{\partial(r v)}{\partial r}= & 0 \\
u \frac{\partial u}{\partial x}+v \frac{\partial u}{\partial r}= & \frac{A}{\rho c} \frac{\partial^{2} u}{\partial r^{2}}+\frac{A}{2 \rho c^{3}}\left(\frac{\partial u}{\partial r}\right)^{2} \frac{\partial^{2} u}{\partial r^{2}}+\frac{A}{r \rho c} \frac{\partial u}{\partial r} \\
& +\frac{A}{6 r \rho c^{3}}\left(\frac{\partial u}{\partial r}\right)^{3}-\sigma \frac{B_{0}^{2}}{\rho} u \\
u \frac{\partial T}{\partial x}+v \frac{\partial T}{\partial r}= & \frac{\dot{\alpha}}{r} \frac{\partial}{\partial r}\left(r \frac{\partial T}{\partial r}\right)+\tau D_{B}\left(\frac{\partial C}{\partial r} \frac{\partial T}{\partial r}\right) \\
& +\frac{\tau D_{T}}{T_{\infty}}\left(\frac{\partial T}{\partial r}\right)^{2}+\frac{A}{c \rho c_{p}}\left(\frac{\partial u}{\partial r}\right)^{2}+\frac{A}{6 c^{3} \rho c_{p}}\left(\frac{\partial u}{\partial r}\right)^{3}
\end{aligned}
$$

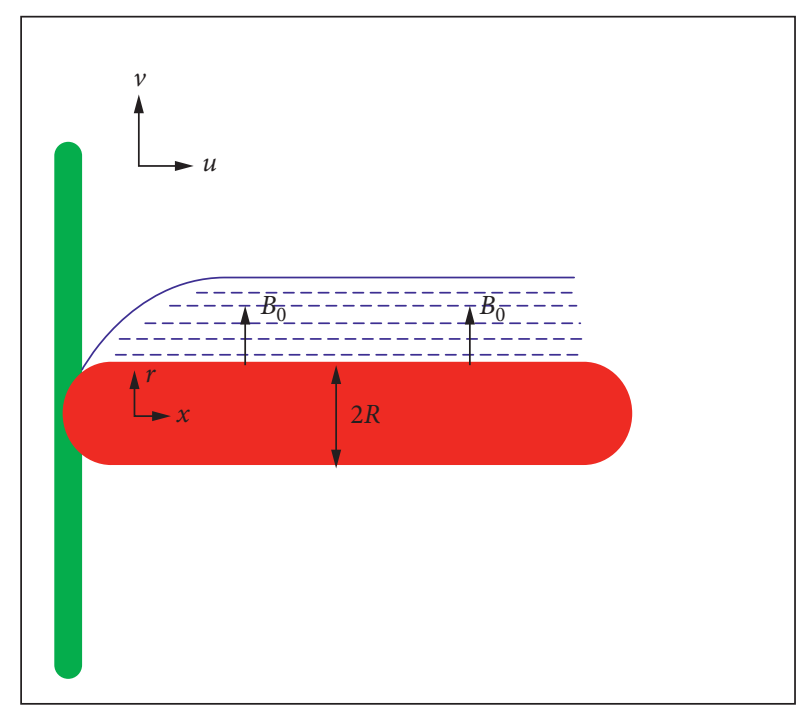

FIGURE 1: Diagram of physical configuration of problem.

$$
\begin{aligned}
u \frac{\partial C}{\partial x}+v \frac{\partial C}{\partial r}= & \frac{D_{B}}{r} \frac{\partial}{\partial r}\left(r \frac{\partial C}{\partial r}\right)+\frac{D_{T}}{r T_{\infty}} \frac{\partial}{\partial r}\left(r \frac{\partial T}{\partial r}\right) \\
& -K\left(C-C_{\infty}\right) .
\end{aligned}
$$

Accompanied to boundary conditions,

$$
\begin{aligned}
u & =U_{w}(x)+L \frac{\partial u}{\partial r}, \\
v & =0, \\
k(T) \frac{\partial T}{\partial r} & =-h_{w}\left[T_{w}-T\right], \\
C & =C_{m}(x), \quad \text { at } r=R, \\
u & \longrightarrow 0, \\
T & \longrightarrow T_{\infty}(x), \\
C & \longrightarrow C_{\infty}, \quad \text { as } r \longrightarrow \infty .
\end{aligned}
$$

In the above equations $\sigma$ is the electrical conductance, $\kappa$ is the thermal conductivity, $R$ is the radius of the cylinder, $\dot{\alpha}=\left(v / \rho c_{p}\right)$ denotes the thermal diffusivity, $c_{p}$ is the specific heat at constant pressure, $D_{B}$ is Brownian motion diffusion coefficient, $D_{T}$ is the thermophoresis diffusion coefficient, $\mathrm{K}$ is chemical reaction rate constant, $U_{w}(x)=a x$ is linear stretching velocity, and wall heat flux coefficient is denoted with $h_{w}$.

The stream function which satisfied the continuity equation is mentioned as follows:

$$
\begin{aligned}
& u=\frac{1}{r} \frac{\partial \psi}{\partial r}, \\
& v=\frac{-1}{r} \frac{\partial \psi}{\partial x} .
\end{aligned}
$$


Equations (4)-(7) can be transformed into nonlinearized ordinary differential equation by employing the following transmutations:

$$
\left.\begin{array}{l}
\eta=\frac{r^{2}-R^{2}}{2 R}\left(\frac{U_{w}}{\nu}\right)^{(1 / 2)} \\
\psi=\left(U_{w} \nu x\right)^{(1 / 2)} R f(\eta) \\
\theta(\eta)=\frac{T-T_{\infty}}{T_{w}-T_{\infty}} \\
\varphi(\eta)=\frac{C-C_{\infty}}{C_{w}-C_{\infty}}
\end{array}\right\} .
$$

After applying the abovementioned transformations, the governing equations, i.e., equations (4)-(7), are transformed into following form:

$$
\begin{aligned}
& \alpha(1+2 \gamma \eta) f^{\prime \prime \prime}+\alpha \beta(1+2 \gamma \eta)^{2} f^{\prime \prime 2} f^{\prime \prime \prime}+\frac{4}{3} \gamma \alpha \beta(1+2 \gamma \eta)\left(f^{\prime \prime}\right)^{3} \\
& +2 \gamma \alpha f^{\prime \prime}-\left(f^{\prime}\right)^{2}+f f^{\prime \prime}-M_{f}^{2} f^{\prime}=0, \\
& (1+2 \gamma \eta) \theta^{\prime \prime}+2 \gamma \theta^{\prime}+\operatorname{Pr} f \theta^{\prime}+\operatorname{Pr}(1+2 \gamma \eta) \\
& \cdot\left(\mathrm{Nb} \theta^{\prime} \phi^{\prime}+\mathrm{Nt} \theta^{\prime 2}\right)=0, \\
& (1+2 \gamma \eta) \phi^{\prime \prime}+2 \gamma \phi^{\prime}+\operatorname{LePr}\left(f \phi^{\prime}-\delta_{c} \phi\right) \\
& +\frac{\mathrm{Nt}}{\mathrm{Nb}}\left(2 r \theta^{\prime}+(1+2 \gamma \eta) \theta^{\prime \prime}\right)=0 .
\end{aligned}
$$
form:

The associated boundary conditions take the following

$$
\begin{aligned}
& f(\eta)=0, \\
& f^{\prime}(\eta)=1+\lambda f^{\prime \prime}(0), \quad \text { at } \eta=0, \\
& f^{\prime}(\eta) \longrightarrow 0, \quad \text { at } \eta \longrightarrow \infty, \\
& \theta^{\prime}(\eta)=-k(1-\theta(\eta)), \quad \text { at } \eta=0, \\
& \theta(\eta) \longrightarrow 0, \quad \text { as } \eta \longrightarrow \infty, \\
& \phi(\eta)=1, \quad \text { at } \eta=0, \\
& \phi(\eta)=0, \quad \text { at } \eta \longrightarrow \infty,
\end{aligned}
$$

where $\alpha$ is the fluid parameter, $\beta$ is the elastic parameter, $\gamma$ is the curvature parameter, $\mathrm{Nb}$ is the Brownian motion parameter, $M_{f}$ is the magnetic strength parameter, $\operatorname{Pr}$ is the Prandtl number, Re is the Reynolds number, Le is the Lewis number, $N t$ is the thermophoresis parameter, and $\delta_{c}$ is the chemical reactive parameter defined as follows:

$$
\begin{aligned}
& \alpha=\frac{A}{\mu c} \\
& \beta=\frac{a^{3} x^{2}}{2 c^{2} v} \\
& \gamma=\frac{1}{R} \sqrt{\frac{\nu}{a}} \\
& \mathrm{Nb}=\frac{\tau D_{B}\left(C_{w}-C_{\infty}\right)}{\rho c_{p} v} \\
& \delta_{c}=\frac{\mathrm{KL}}{a} \\
& M_{f}=\sqrt{\frac{\sigma x B_{0}^{2}}{\rho U_{w}}} \\
& \operatorname{Pr}=\frac{\nu}{\dot{\alpha}} \\
& \operatorname{Re}=\frac{U_{w} x}{v} \\
& \mathrm{Le}=\frac{\dot{\alpha}}{D_{B}} \\
& \mathrm{Nt}=\frac{\tau D_{T}\left(T_{w}-T_{\infty}\right)}{T_{\infty} \rho c_{p} v}
\end{aligned}
$$

The physical parameters of interest, i.e., coefficients of wall drag, wall heat flux, and wall mass flux, are defined as

$$
\begin{gathered}
C_{f}=\frac{\tau_{w}}{(1 / 2) \rho U_{w}^{2}}, \\
N u_{w}=\frac{x q_{w}}{k\left(T_{\infty}-T_{m}\right)}, \\
S h=\frac{x j_{w}}{\left(C_{\infty}-C_{m}\right)},
\end{gathered}
$$

where $\tau_{w}$ is the surface drag force, $q_{w}$ is the surface heat flux, and $j_{w}$ is the surface mass flux given as follows:

$$
\begin{aligned}
& \tau_{w}=\left[\frac{A}{C}\left(\frac{\partial u}{\partial r}\right)+\frac{A}{6 C^{3}}\left(-\frac{\partial u}{\partial r}\right)^{3}\right]_{r=R}, \\
& q_{w}=-k\left(\frac{\partial T}{\partial r}\right)_{r=R}, \\
& J_{w}=-D\left(\frac{\partial C}{\partial r}\right) .
\end{aligned}
$$


Coefficients of wall drag, thermal flux, and mass flux are defined as

$$
\left.\begin{array}{l}
\frac{1}{2} C_{f} \operatorname{Re}^{(-1 / 2)}=\alpha f^{\prime \prime}(\eta)+\frac{\alpha \beta}{3}\left(f^{\prime \prime}(\eta)\right)^{3}, \quad \text { at } \eta \longrightarrow 0 \\
N u_{w} \operatorname{Re}^{(-1 / 2)}=-\theta^{\prime}(\eta), \quad \text { at } \eta \longrightarrow 0 \\
S h \operatorname{Re}^{(-1 / 2)}=-\phi^{\prime}(\eta), \quad \text { at } \eta \longrightarrow 0
\end{array}\right\}
$$

\section{Computational Algorithm}

Shooting technique along with the Runge-Kutta-Fehlberg method (by using Cash and Carp coefficients) is adopted to solve flow-govern problem (Table 1). For this process, flowgovern equations are transformed into

$$
\begin{aligned}
f^{\prime \prime} & =\frac{f^{\prime 2}-f f^{\prime \prime}-2 \gamma \alpha f^{\prime \prime}+M_{f}^{2} f-(4 / 3) \gamma \alpha \beta(1+2 \gamma \eta) f^{\prime \prime 3}}{\left(\alpha(1+2 \gamma \eta)+\alpha \beta(1+2 \gamma \eta)^{2} f^{\prime \prime 2}\right)}, \\
\theta^{\prime \prime} & =\frac{-\left(\operatorname{Pr} f \theta^{\prime}+\operatorname{Pr}(1+2 \gamma \eta)\left(\mathrm{Nb} \theta^{\prime} \phi^{\prime}+\mathrm{Nt} \theta^{\prime 2}\right)+2 \gamma \theta^{\prime}\right)}{(1+2 \gamma \eta)}, \\
\phi^{\prime \prime} & =\frac{-\left(2 \gamma \phi^{\prime}+\operatorname{Le} \operatorname{Pr}\left(f \phi^{\prime}-\delta_{c} \phi\right)+(\mathrm{Nt} / \mathrm{Nb})\left(2 r \theta^{\prime}+(1+2 \gamma \eta) \theta^{\prime \prime}\right)\right)}{(1+2 \gamma \eta)} .
\end{aligned}
$$

By letting a new set of variables,

$$
\begin{aligned}
& e_{1}=f, \\
& e_{2}=f^{\prime}, \\
& e_{3}=f^{\prime \prime}, \\
& e_{4}=\theta, \\
& e_{5}=\theta^{\prime}, \\
& e_{6}=\phi, \\
& e_{7}=\phi^{\prime} .
\end{aligned}
$$

After using new variables, the governing equations take the following form:

$e_{1}^{\prime}=e_{2}$

$e_{2}^{\prime}=e_{3}$,

$e_{3}^{\prime}=\frac{\left(e_{2}\right)^{2}-e_{1} e_{3}-2 \gamma \alpha e_{3}+\left(M_{f}\right)^{2} e_{1}-(4 / 3) \gamma \alpha \beta(1+2 \gamma \eta)\left(e_{3}\right)^{3}}{\left(\alpha(1+2 \gamma \eta)+\alpha \beta(1+2 \gamma \eta)^{2}\left(e_{3}\right)^{2}\right)}$

$e_{4}^{\prime}=e_{5}$
$e_{5}^{\prime}=\frac{-\left(\operatorname{Pr}_{1} e_{5}+\operatorname{Pr}(1+2 \gamma \eta)\left(\mathrm{Nb}_{5} e_{7}+\mathrm{Nt} e_{5}^{2}\right)+2 \gamma e_{3}\right)}{(1+2 \gamma \eta)}$,

$e_{6}^{\prime}=e_{7}$

$e_{7}^{\prime}=\frac{-\left(2 \gamma e_{7}+\operatorname{LePr}\left(e_{1} e_{7}-\delta_{c} e_{6}\right)+(\mathrm{Nt} / \mathrm{Nb})\left(2 \gamma e_{5}+(1+2 \gamma \eta) e_{5}^{\prime}\right)\right)}{(1+2 \gamma \eta)}$.

along boundary conditions

$$
\begin{aligned}
& e_{1}(\eta)=0, \\
& e_{2}(\eta)=1+\lambda e_{3}(\eta), \\
& e_{4}(\eta)=-(1 / k), \\
& e_{5}(\eta)-1, \\
& e_{6}(\eta)=1, \quad \text { at } \eta \longrightarrow 0, \\
& e_{2}(\eta) \longrightarrow 0, \\
& e_{4}(\eta) \longrightarrow 0, \\
& e_{6}(\eta) \longrightarrow 0, \quad \text { as } \eta \longrightarrow \infty .
\end{aligned}
$$

The Runge-Kutta-Fehlberg method is applicable on initial value problem, and in addition, Cash and Karp coefficients are used to raise the accuracy of solution. So, the given boundary conditions are transformed into initial conditions:

$$
\begin{aligned}
& e_{1}(0)=0, \\
& e_{2}(0)=1+\lambda e_{3}(0), \\
& e_{3}(0)=\omega_{1}, \quad \text { (unknown initial condition), } \\
& e_{4}(0)=-\left(\frac{1}{k}\right) e_{5}(0)-1, \\
& e_{5}(0)=\omega_{2}, \quad \text { (unknown initial condition), } \\
& e_{6}(0)=1, \quad \\
& e_{7}(0)=\omega_{3}, \quad \text { (unknown initial condition). }
\end{aligned}
$$

Then, initial value problem is solved and error is computed by comparing given and calculated boundary values. If error is larger than tolerance, i.e., $10^{-6}$, then initial values are modified with Newton's method and process is repeated unless differences are less than $10^{-6}$.

\section{Graphs and Tables}

Impacts and influences of different variables in absence of $\delta_{c}$ are shown in Figures 2-14. 
Table 1: Numerical values of the coefficient given by Cash and Karp.

\begin{tabular}{|c|c|c|c|c|c|c|c|c|}
\hline I & $\mathrm{A}_{i}$ & $B_{i j}$ & $C_{i}$ & $D_{i}$ & & & & \\
\hline 1 & - & - & - & - & - & - & $(37 / 378)$ & $(2825 / 27648)$ \\
\hline 2 & $(1 / 5)$ & $(1 / 5)$ & - & - & - & - & 0 & 0 \\
\hline 3 & $(3 / 10)$ & $(3 / 40)$ & $(9 / 40)$ & - & - & - & $(250 / 621)$ & $(18575 / 48384)$ \\
\hline 4 & $(3 / 5)$ & $(3 / 10)$ & $(-9 / 40)$ & $(6 / 5)$ & - & - & $(125 / 594)$ & (13525/55296) \\
\hline 5 & 1 & $(-11 / 54)$ & $(5 / 2)$ & $(-70 / 27)$ & $(35 / 27)$ & - & 0 & $(277 / 14336)$ \\
\hline 6 & $(7 / 8)$ & $(1631 / 55296)$ & $(175 / 512)$ & $(575 / 13824)$ & $(44275 / 110592)$ & $(253 / 4096)$ & $(512 / 1771)$ & $(1 / 4)$ \\
\hline
\end{tabular}

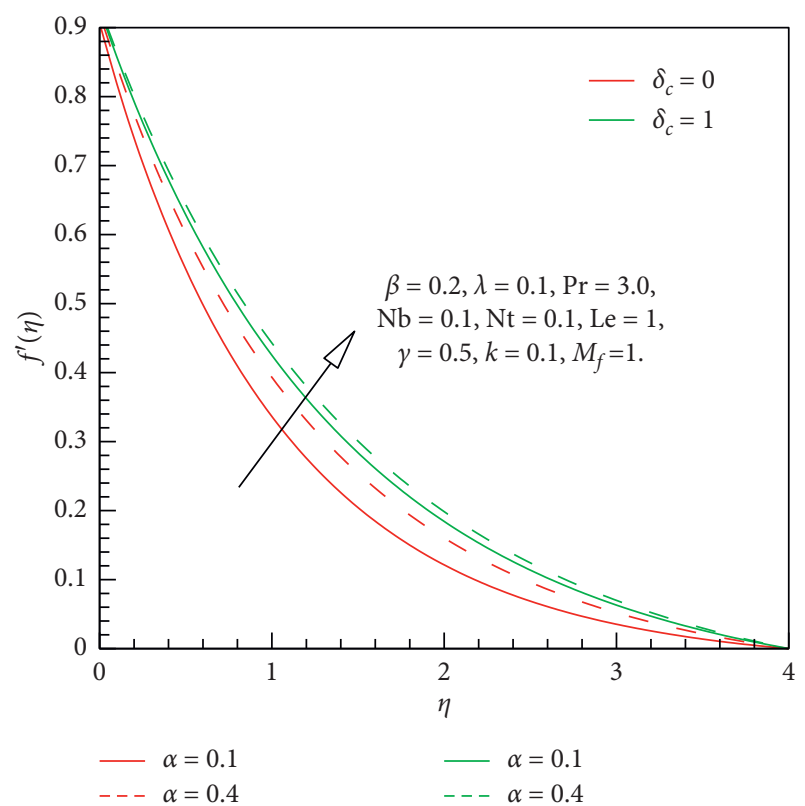

FIGURE 2: Impacts of $\alpha$ on $f^{\prime}(\eta)$ in absence/presence of $\delta_{c}$.

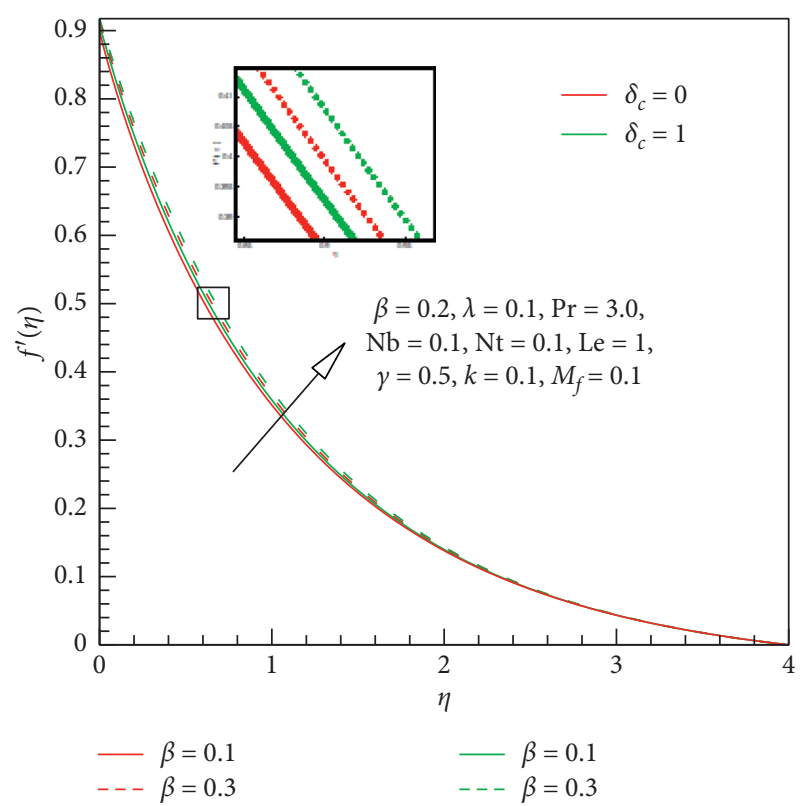

Figure 3: Influence of $\beta$ on $f^{\prime}(\eta)$ in absence/presence of $\delta_{c}$.

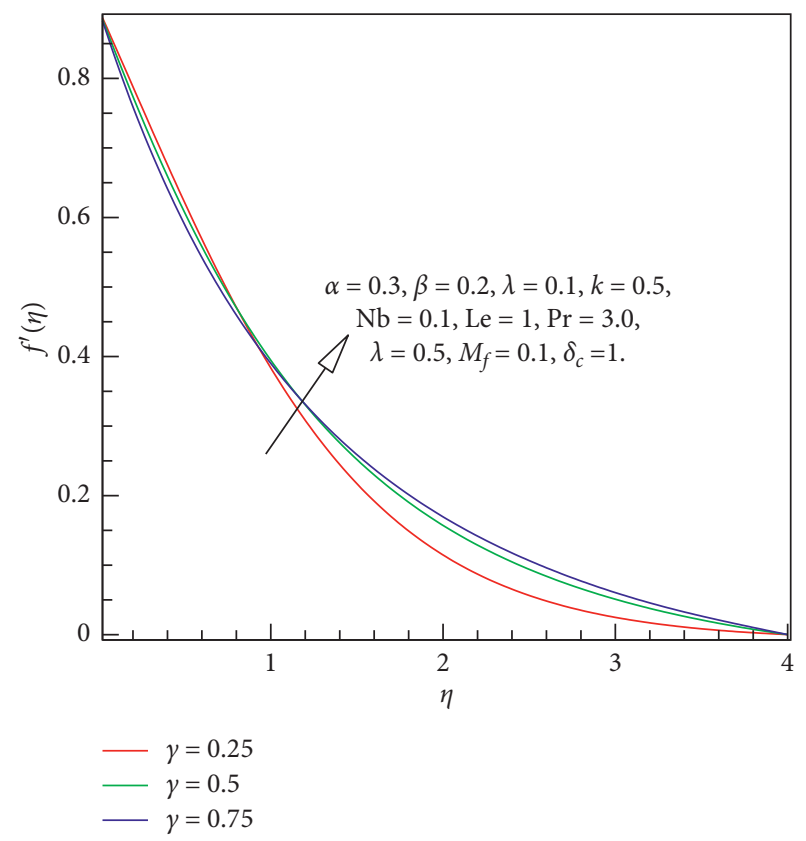

FIgURE 4: $\gamma$ influences on $f^{\prime}(\eta)$ in absence/presence of $\delta_{c}$.

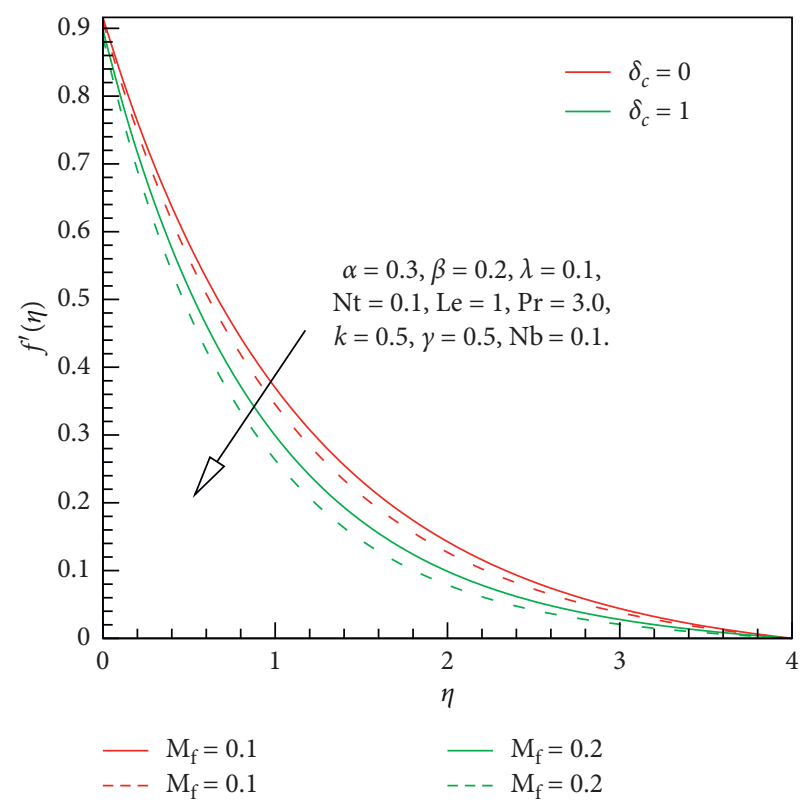

FIgURE 5: $\mathbf{M}_{\mathbf{f}}$ impacts on $f^{\prime}(\eta)$ in absence/presence of $\delta_{\mathbf{c}}$. 


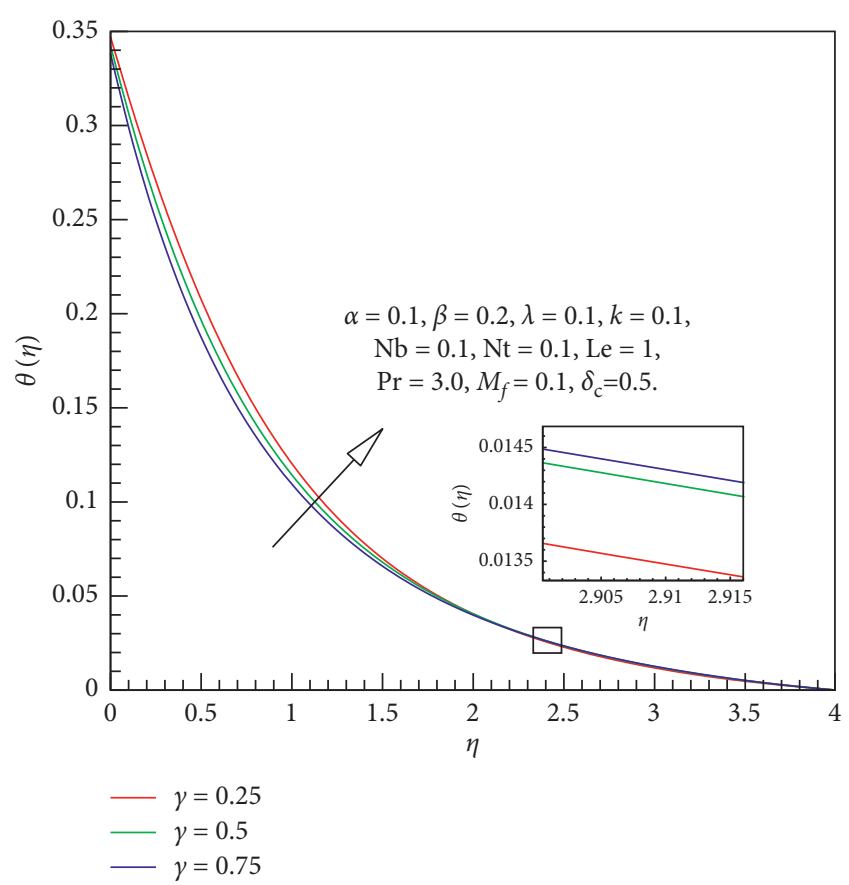

Figure 6: $\gamma$ impacts on $\theta(\eta)$ in the absence/presence of $\delta_{c}$.

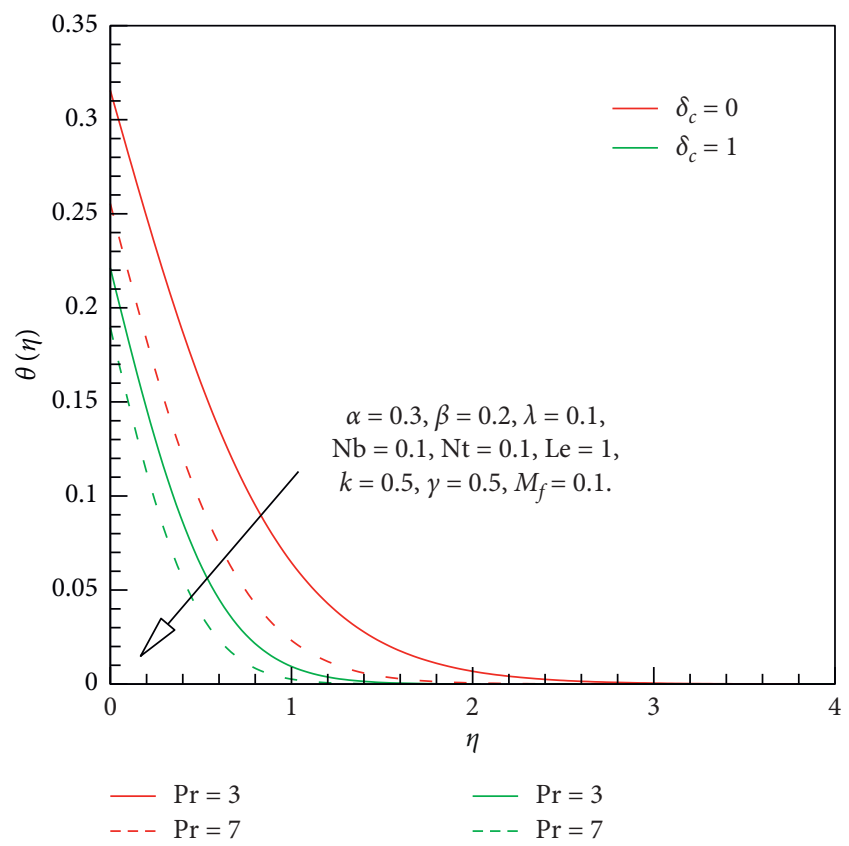

Figure 7: Pr impacts on $\theta(\eta)$ in the absence/presence of $\delta_{c}$.

\section{Straight Line Curve-Fitting Analysis}

The behavior of wall drag, wall heat flux, and wall mass flux is analyzed via straight linearized curved fitting for Prandtl fluid parameter, elastic parameter, curvature parameter, velocity slip, magnetic field, Prandtl number, thermophoretic, Brownian motion, Biot number, Lewis number, and chemical reactive parameters. The Guass and Legendre

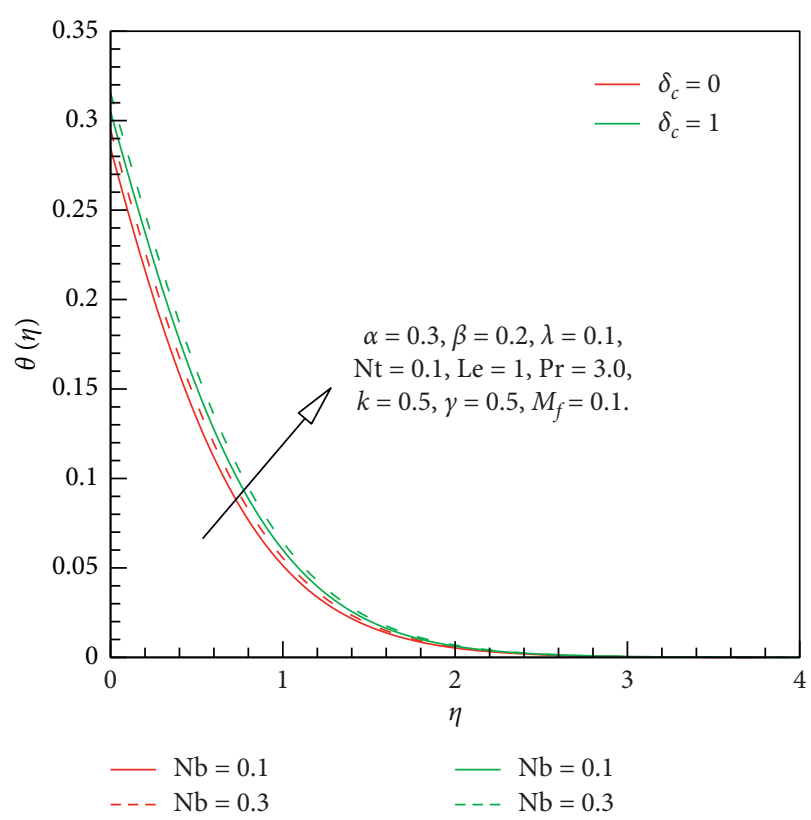

Figure 8: Nb influences on $\theta(\eta)$ in the absence/presence of $\delta_{\mathbf{c}}$.

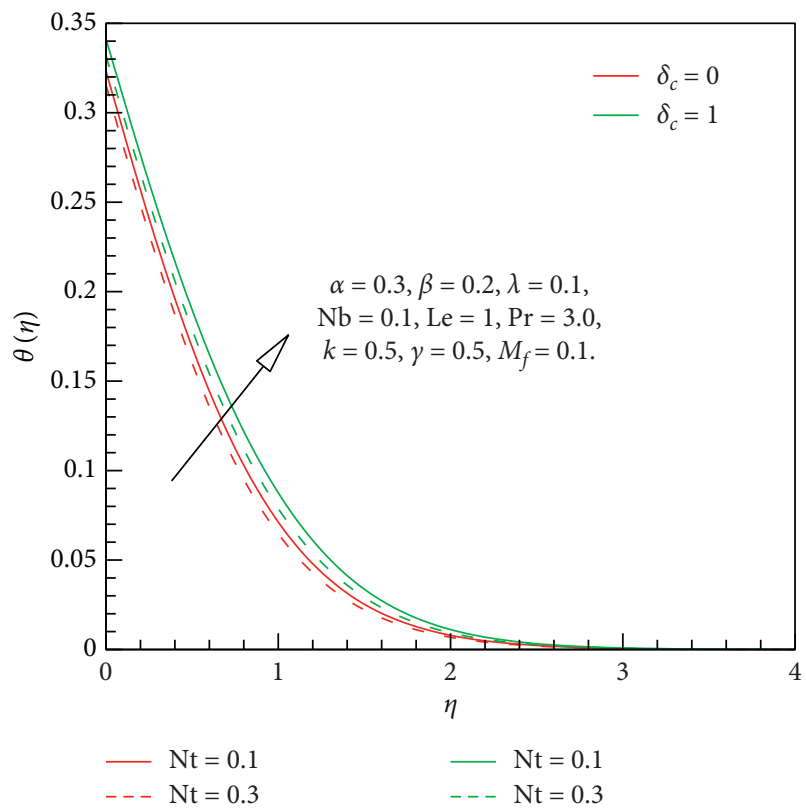

Figure 9: Nt influences on $\theta(\eta)$ in the absence/presence of $\delta_{\mathrm{c}}$.

method was adopted. The expressions for linear approximations are defined such as

$$
\begin{aligned}
n a_{0}+a_{1} \sum \chi_{i} & =\sum \xi_{i}, \\
a_{0} \sum \chi_{i i}+a_{1} \sum \chi_{i}^{2} & =\sum \chi_{i} \xi_{i} .
\end{aligned}
$$

We trace out linearized fitting for wall drag coefficient towards Prandtl fluid, elastic, curvature, velocity slip, and magnetic parameters, i.e., $\alpha, \beta, \gamma, \lambda$, and $M_{f}$. Let $\chi_{i}=(\alpha)_{i}$ and $\xi_{i}=\left(\alpha f^{\prime \prime}(0)+(\alpha \beta / 3)\left(f^{\prime \prime}(0)\right)^{3}\right)_{i}$, then we get 


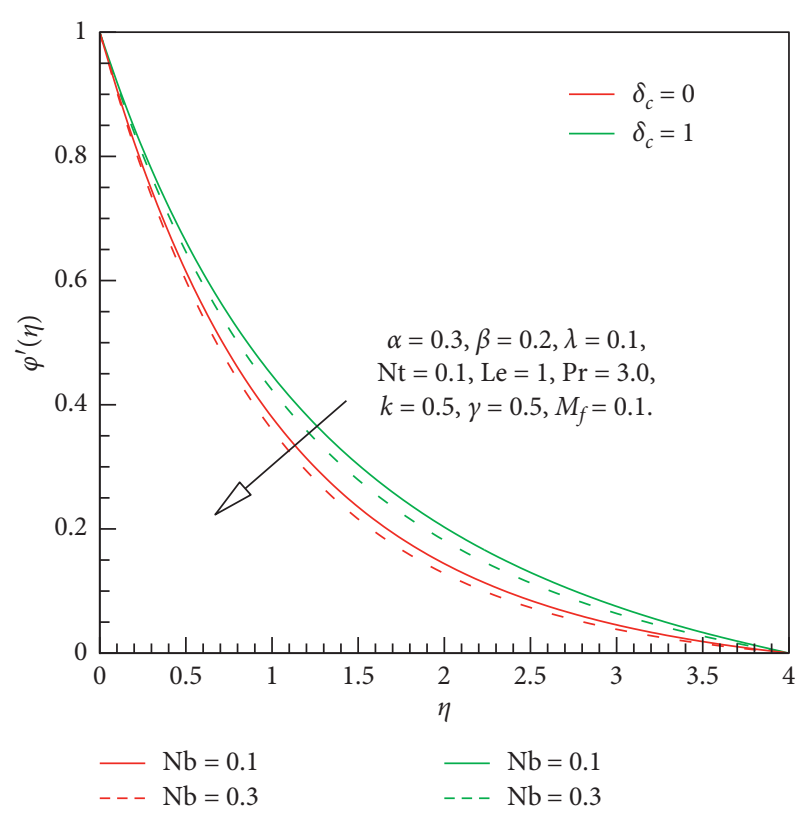

Figure 10: Nb impacts on $\phi(\eta)$ in the absence/presence of $\delta_{\mathbf{c}}$.

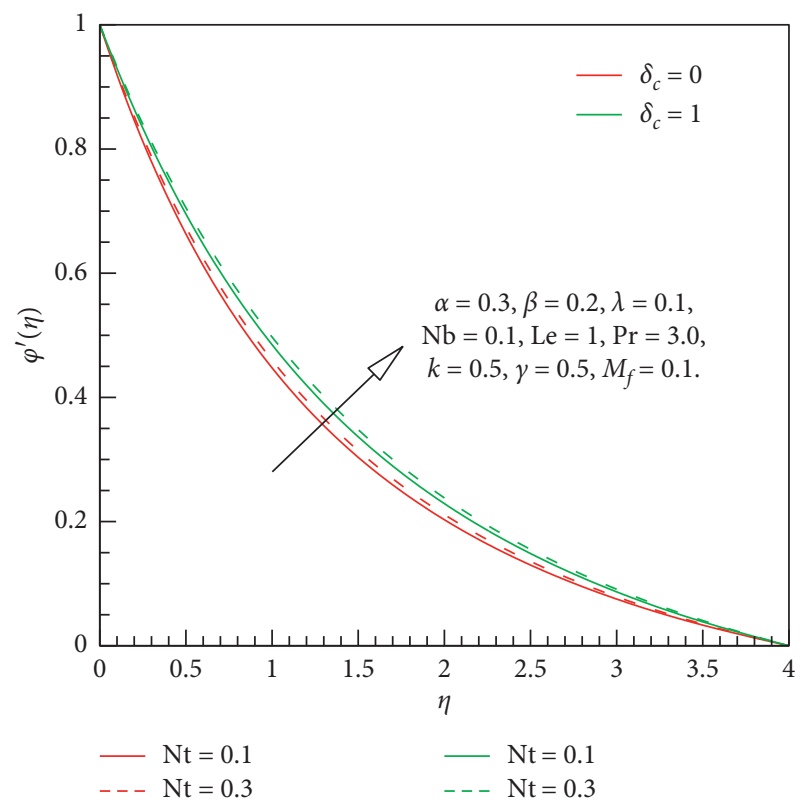

Figure 11: Nt influences on $\phi(\eta)$ in the absence/presence of $\delta_{\mathbf{c}}$.

$$
\begin{aligned}
\sum(\alpha)_{i} & =0.7 \\
\sum(\alpha)_{i}^{2} & =0.25 \\
\sum\left(\alpha f^{\prime \prime}(0)+\frac{\alpha \beta}{3}\left(f^{\prime \prime}(0)\right)^{3}\right)_{i} & =-1.3085 \\
\sum(\alpha)_{i}\left(\alpha f^{\prime \prime}(0)+\frac{\alpha \beta}{3}\left(f^{\prime \prime}(0)\right)^{3}\right)_{i} & =-0.4826 .
\end{aligned}
$$

For straight line approximation, we inserted above expressions in equation (23) and got

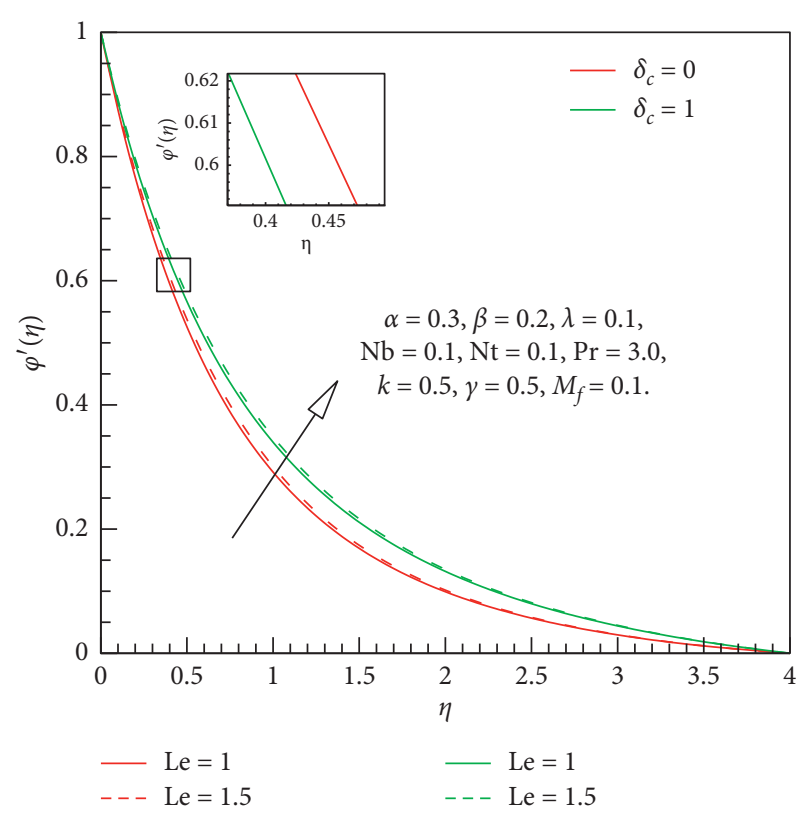

Figure 12: Le influences on $\phi(\eta)$ in the absence/presence of $\delta_{c}$.

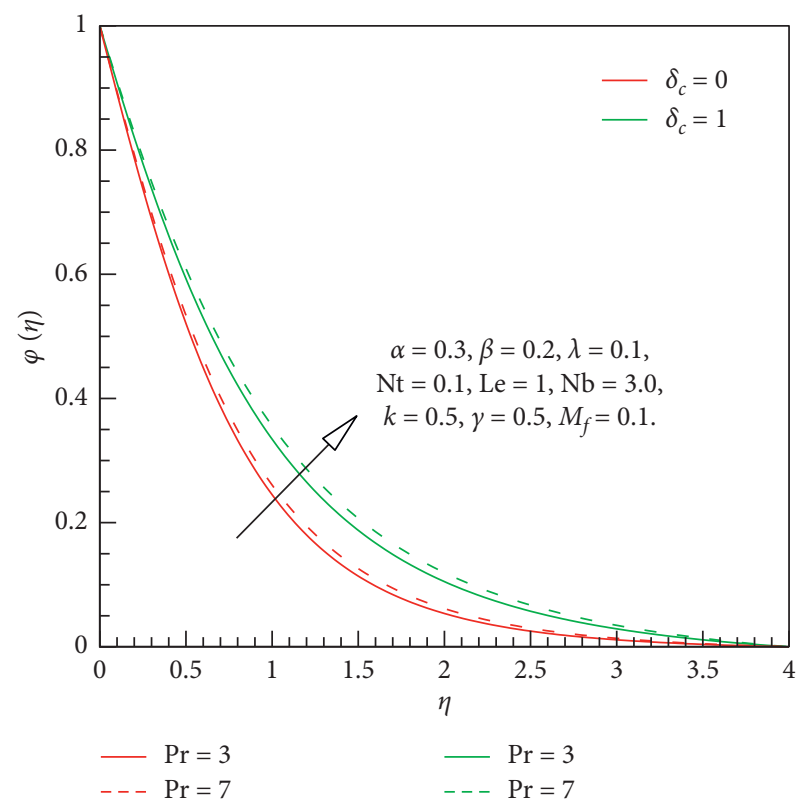

Figure 13: Pr impacts on $\theta(\eta)$ in the absence/presence of $\delta_{\mathbf{c}}$.

$$
\begin{aligned}
2 a_{0}+0.7 a_{1} & =-1.3085 \\
0.7 a_{0}+0.25 a_{1} & =-0.4826
\end{aligned}
$$

By evaluating systemized expression given by (24), we get

$$
\alpha f^{\prime \prime}(0)+\frac{\alpha \beta}{3}\left(f^{\prime \prime}(0)\right)^{3}=a_{0}+a_{1} \alpha
$$

where $a_{0}=1.0694$ and $a_{1}=-4.9249$.

Linearized curve fitting for wall shear stress versus elastic parameter $\beta$ is found such as 


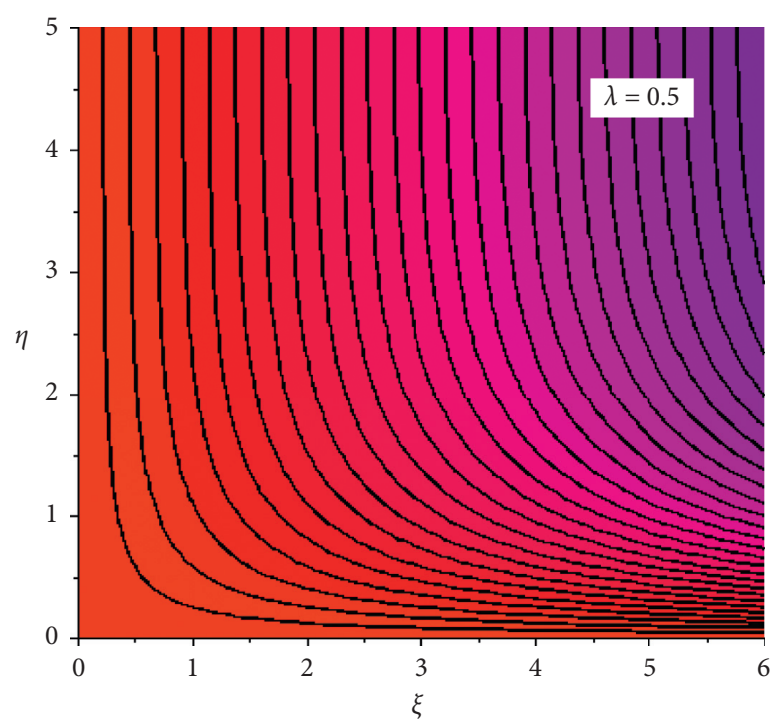

(a)

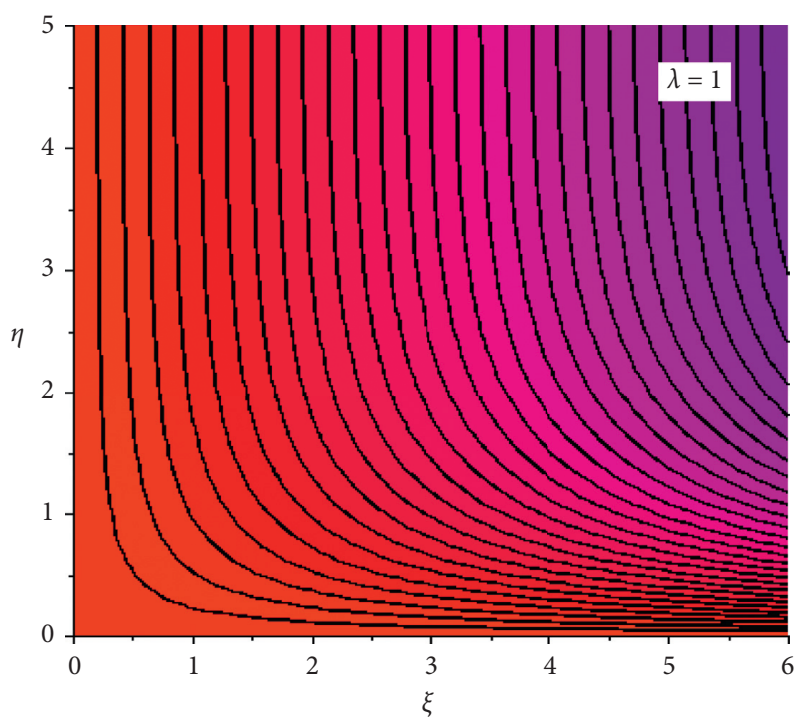

(b)

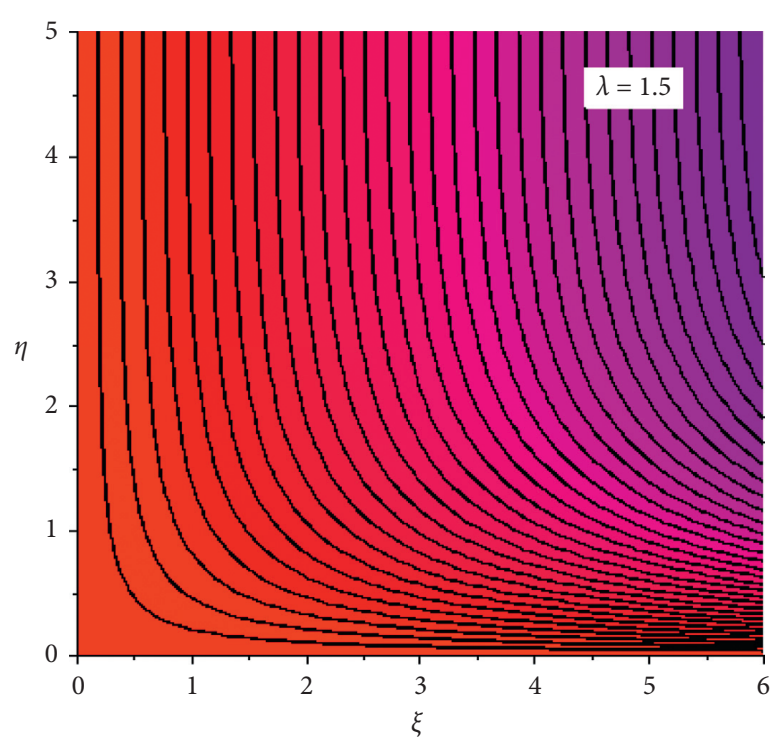

(c)

FIGURE 14: (a) Stream lines against curvature parameter. (b) Stream lines against curvature parameter. (c) Stream lines against curvature parameter.

$$
\begin{aligned}
\sum(\beta)_{i} & =0.7, \\
\sum(\beta)_{i}{ }^{2} & =0.29, \\
\sum\left(\alpha f^{\prime \prime}(0)+\frac{\alpha \beta}{3}\left(f^{\prime \prime}(0)\right)^{3}\right)_{i} & =-1.1708, \\
\sum(\beta)_{i}\left(\alpha f^{\prime \prime}(0)+\frac{\alpha \beta}{3}\left(f^{\prime \prime}(0)\right)^{3}\right)_{i} & =-0.4632, \\
2 b_{0}+0.7 b_{1} & =-1.1708, \\
0.7 b_{0}+0.29 b_{1} & =-0.4632 .
\end{aligned}
$$

The linearized approximation for fluid elastic variable is specified by

$$
\alpha f^{\prime \prime}(0)+\frac{\alpha \beta}{3}\left(f^{\prime \prime}(0)\right)^{3}=P(\beta)=b_{0}+b_{1} \beta,
$$

where $b_{0}=-0.1699$ and $b_{1}=-1.1871$.

The straight line-curved fitted model for the dragged wall number for curvature parameter $\gamma$ is disclosed as follows:

$$
\begin{aligned}
\sum(\gamma)_{i} & =1.5, \\
\sum(\gamma)_{i}{ }^{2} & =1.25, \\
\sum\left(\alpha f^{\prime \prime}(0)+\frac{\alpha \beta}{3}\left(f^{\prime \prime}(0)\right)^{3}\right)_{i} & =-1.5347, \\
\sum(\beta)_{i}\left(\alpha f^{\prime \prime}(0)+\frac{\alpha \beta}{3}\left(f^{\prime \prime}(0)\right)^{3}\right)_{i} & =-1.3299, \\
2 c_{0}+1.5 c_{1} & =-1.5347, \\
1.5 c_{0}+1.25 c_{1} & =-1.3299 .
\end{aligned}
$$


Then, the linear approximations for the curvature parameter are given by

$$
\alpha f^{\prime \prime}(0)+\frac{\alpha \beta}{3}\left(f^{\prime \prime}(0)\right)^{3}=P(\gamma)=c_{0}+c_{1} \gamma,
$$

where $c_{0}=-0.3059$ and $c_{1}=-1.4310$.

Straight line curve fitting for wall drag coefficient against velocity slip parameter $\lambda$ is defined as

$$
\begin{aligned}
\sum(\lambda)_{i} & =0.4, \\
\sum(\lambda)_{i}{ }^{2} & =0.1, \\
\sum\left(\alpha f^{\prime \prime}(0)+\frac{\alpha \beta}{3}\left(f^{\prime \prime}(0)\right)^{3}\right)_{i} & =-0.6685, \\
\sum(\lambda)_{i}\left(\alpha f^{\prime \prime}(0)+\frac{\alpha \beta}{3}\left(f^{\prime \prime}(0)\right)^{3}\right)_{i} & =-0.1191, \\
2 d_{0}+0.4 d_{1} & =-0.6685, \\
0.4 d_{0}+0.1 d_{1} & =-0.1191 .
\end{aligned}
$$

Then, the straight line curves versus the curvature parameter are defined as follows:

$$
\alpha f^{\prime \prime}(0)+\frac{\alpha \beta}{3}\left(f^{\prime \prime}(0)\right)^{3}=P(\lambda)=d_{0}+d_{1} \lambda,
$$

where $d_{0}=-0.4802$ and $d_{1}=0.7300$.

Straight line curve fitting for skin-friction coefficient towards velocity slip parameter $\lambda$ is computed as

$$
\begin{aligned}
\sum\left(M_{f}\right)_{i} & =0.4, \\
\sum\left(M_{f}\right)_{i}^{2} & =0.1, \\
\sum\left(\alpha f^{\prime \prime}(0)+\frac{\alpha \beta}{3}\left(f^{\prime \prime}(0)\right)^{3}\right)_{i} & =-0.9699, \\
\sum\left(M_{f}\right)_{i}\left(\alpha f^{\prime \prime}(0)+\frac{\alpha \beta}{3}\left(f^{\prime \prime}(0)\right)^{3}\right)_{i} & =-0.2095, \\
2 e_{0}+0.4 e_{1} & =-0.9699, \\
0.4 e_{0}+0.1 e_{1} & =-0.2095 .
\end{aligned}
$$

Then, the straight line approximations towards the curvature parameter are given by

$$
\alpha f^{\prime \prime}(0)+\frac{\alpha \beta}{3}\left(f^{\prime \prime}(0)\right)^{3}=P\left(M_{f}\right)=e_{0}+e_{1} M_{f},
$$

where $e_{0}=-0.3297$ and $e_{1}=-0.7759$.

Now, to trace curve-fitted approximation for convectional wall transfer against the curvature parameter, Prandtl number, thermophoresis parameter, Brownian movement parameter, and Biot number are calculated as follows:

$$
\begin{aligned}
\sum(\gamma)_{i} & =1.5, \\
\sum(\gamma)_{i}^{2} & =1.25, \\
\sum\left(-\theta^{\prime}(0)\right)_{i} & =0.5973, \\
\sum(\gamma)_{i}\left(-\theta^{\prime}(0)\right)_{i} & =0.4764, \\
2 f_{0}+1.5 f_{1} & =0.5973, \\
1.5 f_{0}+1.25 f_{1} & =0.4764 .
\end{aligned}
$$

After simplifying, we get

$$
-\theta^{\prime}(0)=P(\gamma)=f_{0}+f_{1} \gamma,
$$

where $f_{0}=0.1281$ and $f_{1}=0.2274$.

Wall heat flux straight line approximation towards the Prandtl number is assessed as

$$
\begin{aligned}
\sum(\operatorname{Pr})_{i} & =3, \\
\sum(\operatorname{Pr})_{i}{ }^{2} & =5, \\
\sum\left(-\theta^{\prime}(0)\right)_{i} & =0.6737, \\
\sum(\operatorname{Pr})_{i}\left(-\theta^{\prime}(0)\right)_{i} & =2.8851, \\
2 g_{0}+8 g_{1} & =0.6737, \\
8 g_{0}+34 g_{1} & =0.4320 .
\end{aligned}
$$

After computing by usual algebraic practice, we attain

$$
-\theta^{\prime}(0)=P(\operatorname{Pr})=g_{0}+g_{1} \operatorname{Pr},
$$

where $g_{0}=4.8624$ and $g_{1}=1.1314$.

Linear approximation for heat transferal rate towards the thermophoresis parameter is calculated by

$$
\begin{aligned}
\sum(\mathrm{Nt})_{i} & =0.1, \\
\sum(\mathrm{Nt})_{i}^{2} & =0.2, \\
\sum\left(-\theta^{\prime}(0)\right)_{i} & =0.4558, \\
\sum(\mathrm{Nt})_{i}\left(-\theta^{\prime}(0)\right)_{i} & =0.0669, \\
2 h_{0}+0.3 h_{1} & =0.4558, \\
0.3 h_{0}+0.05 h_{1} & =0.0669 .
\end{aligned}
$$

After solving by common algebraic practice, we get

$$
-\theta^{\prime}(0)=P(\mathrm{Nt})=h_{0}+h_{1} \mathrm{Nt},
$$


where $h_{0}=4.8624$ and $h_{1}=1.1314$.

Straight line approximation by varying thermophoresis parameter for wall heat flux is found as

$$
\begin{aligned}
\sum(\mathrm{Nb})_{i} & =0.1, \\
\sum(\mathrm{Nb})_{i}{ }^{2} & =0.2, \\
\sum\left(-\theta^{\prime}(0)\right)_{i} & =0.2417, \\
\sum(\mathrm{Nb})_{i}\left(-\theta^{\prime}(0)\right)_{i} & =0.2212, \\
2 j_{0}+0.3 j_{1} & =0.4829, \\
0.3 j_{0}+0.05 j_{1} & =0.0644 .
\end{aligned}
$$

After simplifying, we get

$$
-\theta^{\prime}(0)=P(\mathrm{Nb})=j_{0}+j_{1} \mathrm{Nb} .
$$

where $j_{0}=0.4825$ and $j_{1}=-1.6070$.

Straight line approximations of wall heat flux against the Biot number are found as

$$
\begin{aligned}
\sum(k)_{i} & =0.4, \\
\sum(k)_{i}{ }^{2} & =0.1, \\
\sum\left(-\theta^{\prime}(0)\right)_{i} & =0.2417, \\
\sum(\mathrm{Nb})_{i}\left(-\theta^{\prime}(0)\right)_{i} & =0.2212, \\
2 l_{0}+0.4 l_{1} & =1.2262, \\
0.4 l_{0}+0.1 l_{1} & =0.2257 .
\end{aligned}
$$

After computing by usual algebraic practice, we attain

$$
-\theta^{\prime}(0)=P(k)=l_{0}+l_{1} k,
$$

where $l_{0}=1.0694$ and $l_{1}=-4.9242$.

Wall mass flux straight line approximations versus Lewis number, Prandtl number, thermophoresis parameter, Brownian motion parameter, and chemical reaction parameter are examined as follows:

$$
\begin{aligned}
\sum(\mathrm{Le})_{i} & =1.5, \\
\sum(\mathrm{Le})_{i}^{2} & =1.25, \\
2 m_{0}+1.5 m_{1} & =1.3397, \\
1.5 m_{0}+1.25 m_{1} & =1.0665 .
\end{aligned}
$$

After simplifying, we get

$$
-\phi^{\prime}(0)=P(\mathrm{Le})=m_{0}+m_{1} k,
$$

where $m_{0}=0.2995$ and $m_{1}=0.4938$.

Straight line approximation for surface mass flux for the Prandtl number is calculated as

$$
\begin{aligned}
\sum(\operatorname{Pr})_{i} & =1.5, \\
\sum(\operatorname{Pr})_{i}{ }^{2} & =1.25, \\
\sum\left(-\phi^{\prime}(0)\right)_{i} & =1.1471, \\
\sum(\operatorname{Pr})_{i}\left(-\phi^{\prime}(0)\right)_{i} & =0.8739, \\
2 n_{0}+1.5 n_{1} & =1.2262, \\
1.5 n_{0}+0.1 n_{1} & =0.8739 .
\end{aligned}
$$

After computing by usual algebraic way, we attain

$$
-\phi^{\prime}(0)=P(\operatorname{Pr})=n_{0}+n_{1} \operatorname{Pr},
$$

where $n_{0}=0.4921$ and $n_{1}=1.0847$.

Linear approximation for mass flux towards thermophoresis variable is scrutinized as follows:

$$
\begin{aligned}
\sum(\mathrm{Nt})_{i} & =0.3, \\
\sum(\mathrm{Nt})_{i}{ }^{2} & =0.05, \\
\sum\left(-\phi^{\prime}(0)\right)_{i} & =1.1587, \\
\sum(\mathrm{Nt})_{i}\left(-\phi^{\prime}(0)\right)_{i} & =0.1771, \\
2 q_{0}+0.3 q_{1} & =1.1587, \\
0.3 q_{0}+0.05 q_{1} & =0.1771 .
\end{aligned}
$$

After computing by usual algebraic way, we attain

$$
-\phi^{\prime}(0)=P(\mathrm{Nt})=q_{0}+q_{1} \mathrm{Nt},
$$

where $q_{0}=0.4825$ and $q_{1}=0.6589$.

Surface mass flux straight line approximation for chemical reaction parameter is computed as

$$
\begin{aligned}
\sum\left(\delta_{c}\right)_{i} & =0.3, \\
\sum\left(\delta_{c}\right)_{i}{ }^{2} & =0.05, \\
\sum\left(-\phi^{\prime}(0)\right)_{i} & =1.1505, \\
\sum\left(\delta_{c}\right)_{i}\left(-\phi^{\prime}(0)\right)_{i} & =0.1754, \\
2 s_{0}+0.3 s_{1} & =1.1505, \\
0.3 s_{0}+0.05 s_{1} & =0.1754 .
\end{aligned}
$$

After simplifying, we get

$$
-\phi^{\prime}(0)=P\left(\delta_{c}\right)=s_{0}+s_{1} \delta_{c},
$$

where $s_{0}=0.4905$ and $s_{1}=0.5649$.

\section{Physical Outcomes}

This section is fascinated to examine impact of embedded parameters on fluid concerning profiles. Moreover, physical quantities of interest, i.e., coefficients of wall friction, wall heat flux, and wall mass flux, are reported by means of both straight line curve fitting and tabular structure. In detail, impacts of $\alpha$ and $\beta$ on fluid velocity in the absence/presence of $\delta_{c}$ are presented in Figures 1-3, respectively. From graphical trends, it is seen that both $\alpha$ and $\beta$ cause declination in momentum of fluid flow and associated boundary layer thickness. The reason behind this variation is that with increase in $\alpha$ and $\beta$, viscosity of fluid increases, and hence, movement of fluid decays. It is also divulged that flow regime depicts higher magnitude in the absence of chemical reaction as compared to the presence of chemical reaction. These trends in similar were reported by Eid et al. [14] and Nadeem and Lee [19]. Figure 4 reveals the variation of fluid velocity towards $\gamma$. The fluid velocity accelerated by increasing $\gamma$ (see Figure 4) because larger values of $\gamma$ correspond to reduce 
cylindrical radius which in turn reduces surface area; hence, fluid particles experienced less resistance due to which motion is accelerated. Figure 5 elucidates the impact of $M_{f}$ on velocity. It is clear from these curves that the fluid velocity shows decline trend because an increase in $\mathbf{M}_{\mathbf{f}}$ leads to an increase in Lorentz force and it has tendency to resist the movement of fluid particles, so that velocity decreases. Figure 6 interprets the variations in temperature profile by varying $\gamma$. This figure predicts that the fluid temperature increases by enlarging the curvature parameter. This is due to the fact that when $\gamma$ enlarges, the surface heat flux increases due to which temperature drops adjacent to the cylindrical surface but temperature away from the cylindrical surface enhances. Figure 7 shows impact of Pr on fluid temperature. The graphical plotting depicts decrement in temperature distribution against Pr. It is also noticed that $\delta_{c}=0$ (in absence of chemical reactions), and fluid temperature declines by enlarging the values of $\mathbf{P r}$, but for $\delta_{c}>0$, the decline in fluid temperature is more rapid by enlarging the values of Pr. Figure 8 deliberates the fluctuations in fluid temperature by varying $\mathrm{Nb}$ in the absence/presence of the chemical reaction phenomenon. Since Brownian motion accelerates the particle movement, thus collision process is expedited and hence produces more thermal energy (which can be observed from the graph that fluid temperature rises for larger values of $\mathrm{Nb}$ in both cases). On the other hand, presence of chemical reactive species drops the fluid temperature. Figure 9 shows the temperature profile variations versus $\mathrm{Nt}$ in the absence/presence of chemical reaction. Temperature profile shows increase versus Nt both zero and nonzero $\delta_{\mathrm{c}}$. Figure 10 displays the variations in concentration profile versus combined effect of $\mathrm{Nb}$ and $\delta_{c}$. This graph reveals that the larger values of Nblessen the nanoparticle concentration because enhancement in $\mathrm{Nb}$ corresponds to accelerate random motion of nanoparticles. However, in presence of destructive chemical reaction case, the concentration profile increases. Figure 11 reveals the effect of $\mathrm{Nt}$ in absence and presence of the chemical reaction parameter. It is clear from these curves that both parameters have the same impact on nanoparticle concentration. Figure 12 divulges the influence of Le on nanoparticle volume fraction in absence/presence of chemical reaction. This figure predicts that the concentration profile enlarges versus Le irrespective of absence/presence of chemical reaction. Figure 13 examines the physical significance of $\operatorname{Pr}$ on concentration profile in absence/presence of chemical reaction. It is evident that by increasing $\operatorname{Pr}$ and $\delta_{c}$, nanoparticle volume fraction increases significantly. Figures 14(a)-14(c) are provided to inspect the flow pattern of Prandtl fluid over the stretching cylinder by sketching stream lines against curvature parameter $\gamma$. From stream line plots, it is seen that intensification in magnitude of lines is found with increase in $\gamma$ because as curvature parameter $\gamma$ increases, contact of fluid particles reduces, and as an outcome, less resistance is provided. So, rapid movement in fluid is observed versus $\gamma$. Furthermore, these curves show that yenhances the fluid momentum versus positive alteration in velocity. Figure 15 deliberates variation in skin-friction coefficient against $\alpha, \beta, \gamma, \lambda$, and $\mathbf{M}_{f}$. It is observed that with increase in $\alpha, \beta$, and $\mathbf{M}_{f}$, wall shear stress increases because all of these mentioned parameters uplift the viscosity forces for reduction in velocity whereas reverse pattern is depicted in case of $\lambda$ and $\gamma$. The reason behind increase in skin friction is the uplift of viscosity force in flow domain against these mentioned parameters. Figure 16 displays fluctuations in wall heat flux by varying $\gamma, \mathbf{P r}$ while $\mathbf{N t}, \mathbf{N b}$, and $\mathbf{k}$. It is found that wall heat flux coefficient increases by enhancing $\gamma, \operatorname{Pr}$ while for $\mathbf{N t}, \mathbf{N b}$ and $\mathbf{k}$ reduce. The reason behind enhancement in temperature gradient at wall against $\gamma$ and $\operatorname{Pr}$ is due to the fact that by increasing these parameters, resistance is provided by surface to molecules decrease, and hence, average kinetic energy of molecules increases which raise thermal flux. Figure 17 explicates variation in mass flux against $\mathbf{L e}, \mathbf{P r}, \mathbf{N t}, \mathbf{N b}$, and $\delta_{c}$. It is seen that all mentioned parameters diminish concentration distribution and create motion in fluid molecules, which raises the mass flux with in flow domain. Table 2 delineates the variations in wall friction coefficient versus $\alpha, \beta, \gamma, \lambda$, and, $\mathbf{M}_{f}$ and increasing pattern of wall friction coefficient against $\alpha, \beta, \gamma$ while both $\lambda$ and $\mathbf{M}_{f}$ decrease it. Table 3 is designed to explain the fluctuations in wall heat flux by varying governing parameters. Wall heat flux coefficient increases by enhancing $\gamma, \operatorname{Pr}$ while $\mathbf{N t}, \mathbf{N b}$, and $\mathbf{k}$ reduce it. Table 4 depicts the variations in the local Sherwood number viaLe, $\mathbf{P r}, \mathbf{N t}, \mathbf{N b}$, and $\delta_{c}$, and we observed that with respect to defined parameters, enhancement in the local Sherwood number is manipulated. Tables 5 and 6 provide comparison with the previously published literature and given assurance about collected values in this work.

\section{Key Results}

The current study investigates the magnetic field influences on partial slip flow of Prandtl nanofluid over the stretching cylinder. The fluid flow regime is manifested with the chemical reaction phenomenon. The modelled mathematical equations are reduced in terms of the system of ODEs, and then, the numerical method shooting is implemented to interpret physical aspects of involved parameters. In the presence or absence of the chemical reaction phenomenon, the main findings of present analysis are itemized as follows:

(1) Prandtl fluid velocity reflects an inciting trend towards both Prandtl fluid and elastic parameters

(2) Both fluid velocity and temperature are increasing function of curvature parameter

(3) Magnetic field parameter declines both fluid velocity and temperature

(4) Fluid temperature is increasing function of both Brownian motion and thermophoresis parameters

(5) Nanoparticle concentration shows diminishing variations towards Brownian motion parameter while the opposite trend is noticed versus thermophoresis parameter

(6) Nanoparticle concentration remarks inciting values towards Lewis and Prandtl numbers 


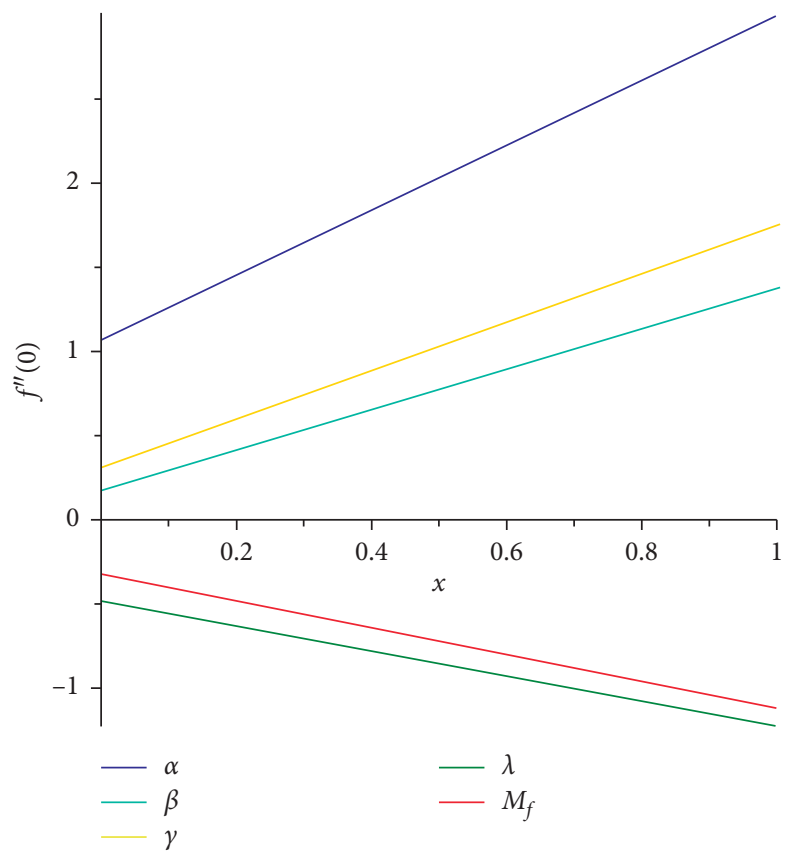

FIGURE 15: Straight line curve fitting for wall shear stress.

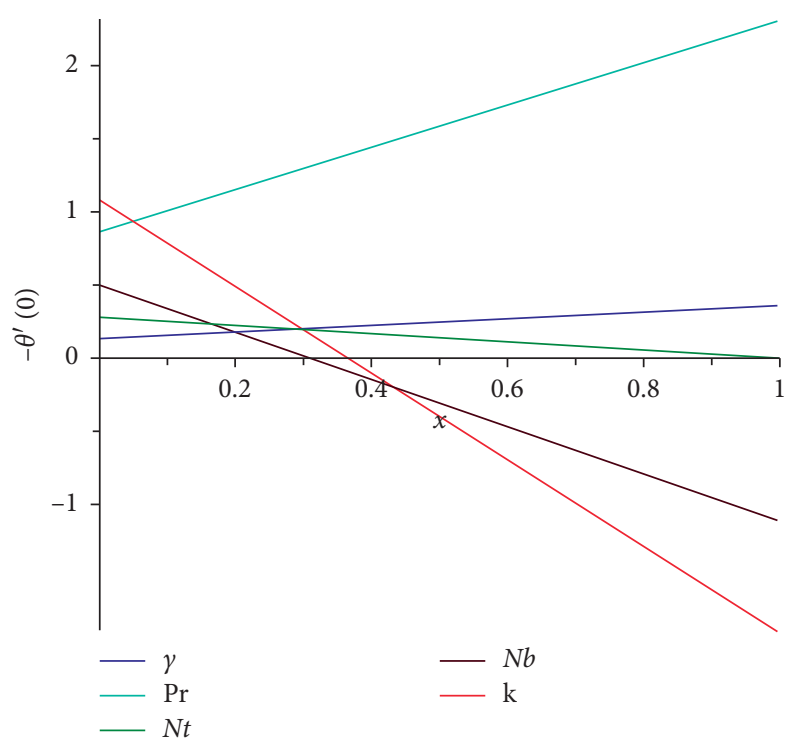

FIGURE 16: Straight line curve fitting for heat transfer rate.

(7) The skin-friction coefficient shows increasing nature for $\alpha, \beta, \gamma$, but opposite behavior is observed for $\lambda$ and $M_{f}$

(8) Wall heat flux coefficient increases against $\gamma$, Pr while it decreases for $\mathrm{Nt}, \mathrm{Nb}$, and $k$

(9) The local Sherwood number shows an inciting value via $\mathrm{Le}, \mathrm{Pr}, \mathrm{Nt}, \mathrm{Nb}$, and $\delta_{c}$

\section{Nomenclature}

$B_{o}: \quad$ Uniform magnetic field

$f^{\prime}(\eta)$ Dimensionless radial velocity profile

$C_{f}: \quad$ Skin-friction coefficient

$\theta(\eta)$ : Dimensionless temperature profile

$A$ and $c$ : Dimensional fluid parameters

$\phi(\eta)$ : Dimensionless concentration profile 


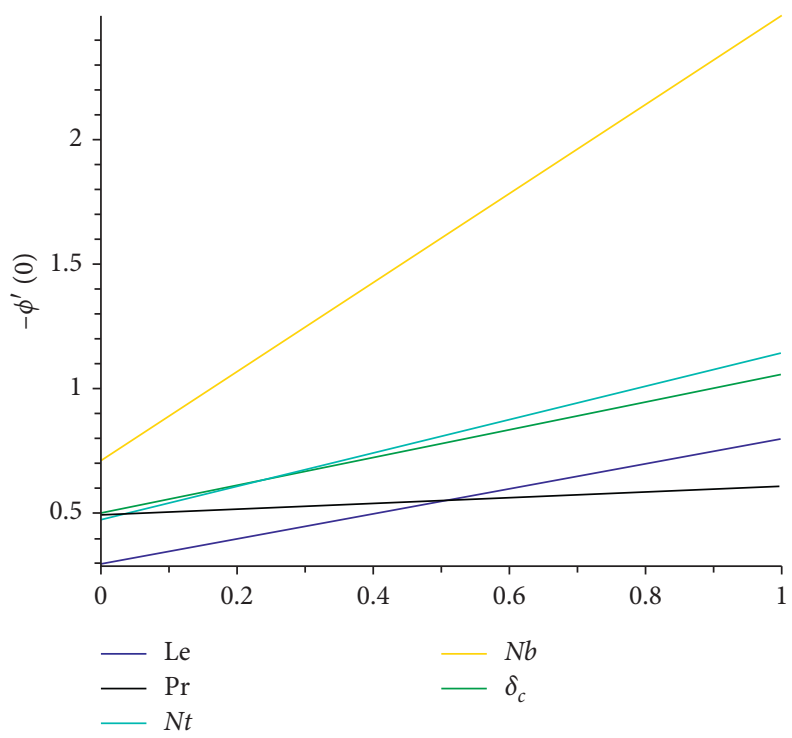

FIGURE 17: Straight line curve fitting for wall mass flux.

TABle 2: Numerical variation of wall friction coefficient for different parameters.

\begin{tabular}{lccccc}
\hline$\alpha$ & $\beta$ & $\gamma$ & $\lambda$ & $\mathbf{M}_{\mathbf{f}}$ & $\alpha f^{\prime \prime}(0)+(\alpha \beta / 3)\left[f^{\prime \prime}(0)\right]^{3}$ \\
\hline 0.3 & 0.2 & 0.5 & - & 0.1 & -0.4073 \\
0.4 & - & - & - & - & -0.9012 \\
0.5 & - & - & - & - & -1.4121 \\
0.3 & 0.2 & - & - & - & -0.4073 \\
- & 0.4 & - & - & - & -0.6481 \\
- & 0.5 & - & - & - & -0.7635 \\
- & 0.2 & -.5 & - & - & -0.4096 \\
- & - & 1.5 & - & - & -1.1251 \\
- & - & 0.5 & - & - & -1.7481 \\
- & - & - & 0.1 & -0.4073 \\
- & - & - & 0.5 & - & -0.2612 \\
- & - & - & 0.1 & 0.1 & -0.1815 \\
- & - & - & - & 0.3 & -0.4073 \\
- & - & - & - & 0.7 & -0.5626 \\
- & - & - & & -0.2253 \\
\hline
\end{tabular}

TABLE 3: Numerical variation of HTR for different parameters.

\begin{tabular}{|c|c|c|c|c|c|}
\hline$\gamma$ & Pr & $\mathrm{Nt}$ & $\mathrm{Nb}$ & $\mathrm{k}$ & $-\theta^{\prime}(0)$ \\
\hline 0.5 & 3 & 0.1 & 0.1 & 0.5 & 0.2417 \\
\hline 1 & - & - & - & - & 0.3556 \\
\hline 1.5 & - & - & - & - & 0.4517 \\
\hline 0.5 & 3 & - & - & - & 0.2417 \\
\hline- & 5 & - & - & - & 0.4320 \\
\hline- & 7 & - & - & - & 0.7121 \\
\hline- & 3 & 0.1 & - & - & 0.2417 \\
\hline- & - & 0.2 & - & - & 0.2141 \\
\hline- & - & 0.3 & - & - & 0.1782 \\
\hline- & - & 0.1 & 0.1 & - & 0.2417 \\
\hline- & - & - & 0.2 & - & 0.2212 \\
\hline- & - & - & 0.3 & - & 0.2031 \\
\hline - & - & - & 0.1 & 0.1 & 0.7104 \\
\hline- & - & - & - & 0.3 & 0.5158 \\
\hline- & - & - & - & 0.7 & 0.1715 \\
\hline
\end{tabular}


TABLE 4: Numerical variation of MTR for different parameters.

\begin{tabular}{|c|c|c|c|c|c|}
\hline Le & Pr & $\mathrm{Nt}$ & $\mathrm{Nb}$ & $\delta_{c}$ & $-\phi^{\prime}(0)$ \\
\hline 0.5 & 0.5 & 0.1 & 0.1 & 0.1 & 0.5463 \\
\hline 1 & - & - & - & - & 0.7934 \\
\hline 1.5 & - & - & - & - & 0.9946 \\
\hline 0.5 & 0.5 & - & - & - & 0.5463 \\
\hline- & 1 & - & - & - & 0.6008 \\
\hline - & 1.5 & - & - & - & 0.6458 \\
\hline - & 0.5 & 0.1 & - & - & 0.5463 \\
\hline - & - & 0.2 & - & - & 0.6124 \\
\hline - & - & 0.3 & - & - & 0.7314 \\
\hline - & - & 0.1 & 0.1 & - & 0.5463 \\
\hline- & - & - & 0.2 & - & 0.8201 \\
\hline - & - & - & 0.3 & - & 1.1241 \\
\hline - & - & - & - & 0.1 & 0.5463 \\
\hline- & - & - & - & 0.2 & 0.6042 \\
\hline- & - & - & - & 0.3 & 0.6513 \\
\hline
\end{tabular}

TABLE 5: Comparison of wall shear stress by varying magnetic field parameter $\mathbf{M}_{\mathbf{f}}$ while considering other parameters to zero.

\begin{tabular}{lccc}
\hline $\mathbf{M}_{\mathbf{f}}$ & Akbar at el. [32] & Malik at el. [33] & Present \\
\hline 0.0 & -1 & -1 & -1 \\
0.5 & -1.11803 & -1.11803 & -1.1180 \\
1.0 & -1.41421 & -1.41419 & -1.4137 \\
5.0 & -2.44949 & -2.44945 & -2.4495 \\
10 & -3.31663 & -3.31657 & -3.3166 \\
100 & -10.04988 & -10.04981 & -10.0500 \\
500 & -22.38303 & -22.38294 & -22.3835 \\
1000 & -31.63859 & -31.63851 & -31.6391 \\
\hline
\end{tabular}

TABLE 6: Comparison of results for the reduced Nusselt number $-\theta^{\prime}(0)$ against $\operatorname{Pr}$ and considering all other parameters zero.

\begin{tabular}{lccc}
\hline Pr & Present results & Wang [30] & Gorla and Sidawi [31] \\
\hline 0.07 & 0.0663 & 0.0656 & 0.0656 \\
0.20 & 0.1619 & 0.1619 & 0.1619 \\
0.70 & 0.4539 & 0.4539 & 0.4539 \\
2.00 & 0.9113 & 0.9114 & 0.9114 \\
7.00 & 1.8954 & 1.8954 & 1.9805 \\
20.00 & 3.3539 & 3.3539 & 3.3539 \\
70.00 & 6.4621 & 6.4622 & 6.4622 \\
\hline
\end{tabular}

$A_{1}: \quad$ First Rivlin-Ericksen tensor

$v$ : $\quad$ Kinematic viscosity

M: $\quad$ Magnetic parameter

$\mu$ : Dynamic viscosity

Pr: $\quad$ Prandtl number

$\rho: \quad$ Density

r, $x$ : Cylindrical coordinate system

$\rho C_{P}$ : Volumetric heat capacity

$K: \quad$ Dimensionless chemical reaction parameter

$\gamma: \quad$ Curvature parameter

$\alpha$ : $\quad$ Fluid parameter

$\beta$ : $\quad$ Elastic parameter

$\mathrm{Nu}$ : Nusselt number

$\mathrm{Nb}$ : Brownian motion parameter
Nt: Thermophoresis parameter

$\delta_{c}$ : Dimensionless chemical reaction parameter

\section{Greek symbols}

$\dot{\alpha}$ : Thermal diffusivity $\left[\mathrm{m}^{2} / \mathrm{s}\right]$

$\eta$ : Independent similarity variable.

\section{Data Availability}

The data used to support the findings of this study are available from the corresponding author after acceptance of publication.

\section{Conflicts of Interest}

The authors declare that they have no conflicts of interest.

\section{Acknowledgments}

The authors extend their appreciation to the Deanship of Scientific Research at Majmaah University for funding this work under project number RGP-2019-3.

\section{References}

[1] M. W. Dunn, "Non-newtonian fluid flow through fabrics: national textile center," Annual Report, vol. M98-P02, p. 1, 1999. 
[2] S. Nadeem, H. Sadaf, and N. S. Akbar, "Analysis of peristaltic flow of prandtl fluid model in an endoscope," Journal of Power Technology, vol. 94, pp. 1-11, 2014.

[3] N. S. Akbar, "Blood flow analysis of prandtl fluid model in tapered stenosed arteries," Ain Shams Engineering Journal, vol. 5, no. 4, pp. 1267-1275, 2014.

[4] S. Jothi, A. R. Parasad, and M. V. S. Reddy, "Perestaltic flow of prandtl fluid in a symmetric channel under the effect of magnetic field," Advances in Applied Science Research, vol. 3, pp. 2108-2119, 2012.

[5] S. Nazari, R. Ellahi, M. M. Sarafraz, M. R. Safaei, A. Asgari, and O. A. Akbari, "Numerical study on mixed convection of a non-newtonian nanofluid with porous media in a two liddriven square cavity," Journal of Thermal Analysis and Calorimetry, vol. 140, no. 3, pp. 1121-1145, 2020.

[6] A. F. A. Hossainy, M. R. Eid, and M. S. Zoromba, "SQLM for external yield stress effect on 3D MHD nanofluid flow in a porous medium," Physica Scripta, vol. 94, p. 10, 2019.

[7] S. Bilal, K. U. Rehman, M. Y. Malik, A. Hussain, and M. Awais, "Effect logs of double diffusion on MHD prandtl nano fluid adjacent to stretching surface by way of numerical approach," Results in Physics, vol. 7, pp. 470-479, 2017.

[8] S. P. Anjalidevi and R. Kandasamy, "Effects of chemical reaction, heat and mass transfer on laminar flow along a semi infinite horizontal plate," Heat and Mass Transfer, vol. 35, no. 6, pp. 465-467, 1999.

[9] Y. Zhang and L. Zheng, "Analysis of MHD thermosolutal marangoni convection with the heat generation and a firstorder chemical reaction," Chemical Engineering Science, vol. 69, no. 1, pp. 449-455, 2012.

[10] M. A. Chaudhary and J. H. Merkin, "A simple isothermal model for homogeneous-heterogeneous reactions in boundary-layer flow. I equal diffusivities," Fluid Dynamics Research, vol. 16, no. 6, pp. 311-333, 1995.

[11] R. Nandkeolyar, S. S. Motsa, and P. Sibanda, "Viscous and joule heating in the stagnation point nanofluid flow through a stretching sheet with homogenous-heterogeneous reactions and nonlinear convection," Journal of Nanotechnology in Engineering and Medicine, vol. 4, no. 4, 2013.

[12] A. F. A. Hossainy and M. R. Eid, "Structure, DFT calculations and heat transfer enhancement in $[\mathrm{ZnO} / \mathrm{PG}+\mathrm{H} 2 \mathrm{O}] \mathrm{C}$ hybrid nanofluid flow as a potential solar cell coolant application in a double-tube," Journal of Materials Science: Materials in Electronics, vol. 31, pp. 15243-15257, 2020.

[13] M. R. Eid and O. D. Makinde, "Solar radiation effect on a magneto nanofluid flow in a porous medium with chemically reactive species," International Journal of Chemical Reactor Engineering, vol. 16, pp. 2017-0212, 2018.

[14] M. R. Eid, K. L. Mahny, A. Dar, and T. Muhammad, "Numerical study for carreau nanofluid flow over a convectively heated nonlinear stretching surface with chemically reactive species," Physica A: Statistical Mechanics and Its Applications, vol. 540, p. 123063, 2020.

[15] L. Zhang, M. M. Bhatti, and E. E. Michaelides, "Entropy generation in magnetized blood flow through a finite wavy channel under slip conditions," Journal of Non-equilibrium Thermodynamics, vol. 45, no. 4, p. 419, 2020.

[16] M. M. Bhatti, A. Riaz, L. Zhang, S. M. Sait, and R. Ellahi, "Biologically inspired thermal transport on the rheology of williamson hydromagnetic nanofluid flow with convection: an entropy analysis," Journal of Thermal Analysis and Calorimetry, 2020.
[17] C. SU, "Enhancing thermal conductivity of fluids with nanoparticles," ASME-Publications, vol. 231, pp. 99-106, 1995.

[18] J. Buongiorno, "Convective transport in nanofluids," Journal of Heat Transfer, vol. 128, no. 3, pp. 240-250, 2006.

[19] S. Nadeem and C. Lee, "Boundary layer flow of nanofluid over an exponentially stretching surface," Nanoscale Research Letters, vol. 7, no. 1, p. 7, 2012.

[20] M. Raza, R. Ellahi, S. M. Sait, M. M. Sarafraz, M. S. Shadloo, and I. Waheed, "Enhancement of heat transfer in peristaltic flow in a permeable channel under induced magnetic field using different CNTs," Journal of Thermal Analysis and Calorimetry, vol. 140, no. 3, pp. 1277-1291, 2020.

[21] S. M. Peyghambarzadeh, S. H. Hashemabadi, M. S. Jamnani, and S. M. Hoseini, "Improving the cooling performance of automobile radiator with $\mathrm{Al}_{2} \mathrm{O}_{3}$ /water nanofluid," Applied Thermal Engineering, vol. 31, no. 10, pp. 1833-1838, 2011.

[22] A. A. Alaidrous and M. R. Eid, "3D electromagnetic radiative non-newtonian nanofluid flow with Joule heating and higherorder reactions in porous materials," Scientific Reports, vol. 10, no. 4, pp. 41598-42020, 2020.

[23] M. R. Eid and F. Mabood, "Entropy analysis of a hydromagnetic micropolar dusty carbon NTs-kerosene nanofluid with heat generation: darcy-forchheimer scheme," Journal of Thermal Analysis and Calorimetry, 2020.

[24] M. R. Eid, "Effects of NP shapes on non-newtonian bionanofluid flow in suction/blowing process with convective condition: sisko model," Journal of Non-equilibrium Thermodynamics, vol. 45, 2020.

[25] W. A. Khan and I. Pop, "Boundary-layer flow of a nanofluid past a stretching sheet," International Journal of Heat and Mass Transfer, vol. 53, no. 11-12, pp. 2477-2483, 2010.

[26] L. A. Khan, M. Raza, N. A. Mir, and R. Ellahi, "Effects of different shapes of nanoparticles on peristaltic flow of MHD nanofluids filled in an asymmetric channel," Journal of Thermal Analysis and Calorimetry, vol. 140, no. 3, pp. 879890, 2020.

[27] A. Ahmed, "Flow of ReinerPhilippoff based nano-fluid past a stretching sheet," Journal of Molecular Liquids, vol. 219, pp. 643-646, 2016.

[28] C. Y. Wang, "Free convection on a vertical stretching surface," ZAMM - Journal of Applied Mathematics and Mechanics/ Zeitschrift für Angewandte Mathematik und Mechanik, vol. 69, no. 11, pp. 418-420, 1989.

[29] R. S. R. Gorla and I. Sidawi, "Free convection on a vertical stretching surface with suction and blowing," Applied Science Research, vol. 52, pp. 247-257, 1994.

[30] M. R. Eid, A. F. A. Hossainy, and M. S. Zoromba, "FEM for blood-based SWCNTs flow through a circular cylinder in a porous medium with electromagnetic radiation," Communications in Theoretical Physics, vol. 71, p. 12, 2019.

[31] Lahmar, "Examined the fluid flow and thermal transportation of an unsteady nanofluid (fe3o4-water) between two plates parallel to each other, observing the case where the thermal heat conduction is a function of temperature in the presence of magnetic field," 2020.

[32] N. S. Akbar, A. Ebai, and Z. H. Khan, "Numerical analysis of magnetic field effects on eyring-powell fluid flow towards a stretching sheet," Journal of Magnetism and Magnetic Materials, vol. 382, pp. 355-358, 2015.

[33] M. Y. Malik, T. Salahuddin, A. Hussain, and S. Bilal, "MHD flow of tangent hyperbolic fluid over a stretching cylinder: using keller box method," Journal of Magnetism and Magnetic Materials, vol. 395, pp. 271-276, 2015. 